\title{
Conducting Comprehensive Environmental Scan in Health System \& Policy Research: A Process for Assessing the Subject Matter Landscape
}

\author{
Maaz Shahid ${ }^{1,2}$ and Tanvir C Turin ${ }^{1,3,4, *}$ \\ ${ }^{1}$ Department of Family Medicine, Cumming School of Medicine, University of Calgary, Calgary, AB, \\ Canada \\ ${ }^{2}$ Department of Epidemiology, Biostatistics and Occupational Health, McGill University, Montreal, \\ Quebec, Canada. \\ ${ }^{3}$ Department of Community Health Sciences, Cumming School of Medicine, University of Calgary, \\ Calgary, AB, Canada \\ ${ }^{4}$ O'Brien Institute for Public Health, University of Calgary, Calgary, AB, Canada \\ "Corrresponding author: chowdhut@ucalgary.ca
}

Received: March 2, 2018; revised: June 8, 2018; accepted: July 31, 2018.

\begin{abstract}
Environmental scans provide researchers with an assessment of the landscape around an issue of interest. In this process relevant information is systematically amassed to identify current status, scopes or opportunities, and risks. This paper aims to serve as a basic and surface level guide to understanding and planning for conducting an environmental scan. The intended audience includes students and researchers new to the use of environmental scans. Before discussion of all the steps, some examples of the use of environmental scans in health research is provided. The process of conducting an environmental scan is outlined in five steps that revolve around purpose, people, questions, information gathering and presenting. The paper concludes with a discussion on advantages and challenges of conducting environmental scans.
\end{abstract}

Keywords: Environmental scan, health service research, health policy research, knowledge synthesis, stakeholder. 


\section{Introduction}

With more and more information being accessible due to the continuous digitalization of healthcare data and findings, health researchers need a way to filter and assess this constant stream of information. This is critical when looking to assess the working environment around a particular health issue, since doing so allows researchers to build on one another's work, instead of simply reinventing the wheel. This in turn will set up the program of research being built on a strong platform of existing knowledge and lead towards sustainability. There is much work to be done in this regard, as researchers often fail to cite previous work in their study area (Glasziou et al., 2014). One way to increase the recognition of previous work and identify the current status of an issue is through processes such as environmental scans. It is important to note that environmental scans are different from literature reviews in that a literature review is an important aspect of a successful scan, among other components. A comprehensive environmental scan will assist researchers in contextualizing and focusing their research program. This paper provides a surface level guide to executing an environmental scan within the context of health research. The intended audience includes academic researchers, graduate students, community organization members, and citizen researchers new to the use of environmental scans.

\subsection{What is an Environmental Scan?}

The exact definition of an environmental scan varies with the context for which it is used, as seen by various researchers and organizations having their own definitions, presented in Table 1. Since our objective was to contextualize in the backdrop of health research, we present an environmental scan as a process or tool used to assess the working environment around and within a particular health issue.

Table 1: Various Definitions of an Environmental Scan.

\begin{tabular}{|c|c|}
\hline Source & Definition \\
\hline Choo et al. (2001) & $\begin{array}{l}\text { "Acquisition and use of information about events, trends, and re- } \\
\text { lationships in an organization's external environment, the knowl- } \\
\text { edge of which would assist management in planning the organi- } \\
\text { zation's future course of action" }\end{array}$ \\
\hline Morrison (1992) & $\begin{array}{l}\text { ".... a method that enables decisionmakers both to understand } \\
\text { the external environment and the interconnections of its various } \\
\text { sectors and to translate this understanding into the institution's } \\
\text { planning and decisionmaking processes." }\end{array}$ \\
\hline $\begin{array}{l}\text { Canadian Agency for } \\
\text { Drugs and Technologies } \\
\text { in Health (May, 2015) }\end{array}$ & $\begin{array}{l}\text { "Inform decision-makers about the use of health technologies } \\
\text { across jurisdictions, particularly with respect to practice variation } \\
\text { and policy gaps" }\end{array}$ \\
\hline
\end{tabular}

\&RF WWw.jBiomedAnalytics.org 


\subsection{Why are Environmental Scans Used?}

Originally used by organizations and businesses to assess the internal and external factors affecting an organization (Graham et al., 2008), studies show an environmental scan to be a highly effective tool that is employed by the successful businesses and absent in the businesses that failed (Choo et al., 2001; Albright, 2004). Similarly, health organizations and policy makers would do well to incorporate environmental scans when assessing their current environment, planning for future challenges, and to take advantage of their strengths.

In the context of increasing health research findings and implementation, environmental scans comprise the first of three steps that are generally used to explain public health assessment (Rowel et al., 2005), followed by policy development and assurance. One of the many benefits of using an environmental scan to assess the environment is that the assessment can be tailored specifically to the resources available and the people or communities involved, which results in increased efficiency and probability of success.

\subsection{Examples of Environmental Scans}

Environmental scans can be done in many different contexts. Following are three papers that used environmental scans, in different contexts. Rowel et al. (2005) conducted an environmental scan in the first phase of a project designed to increase cancer screenings among African Americans in Baltimore, Maryland, USA - a group that is disproportionately affected by cancer (Rowel et al., 2005). This scan comprised of eight methods of collecting data, all of which were unpublished sources and either professional or lay viewpoints. From the collected data, the researchers made five key conclusions, among them being able to understand issues around cancer screening from both the community's and service providers' perspectives. These results were then used for implementing a pilot prostate cancer screening project, keeping in mind the five key conclusions mentioned above.

Aslakson et al. (2014) published a protocol for conducting an environmental scan to evaluate the possible addition of healthcare aids that can assist patients who are about to undergo high-risk surgery with advanced care planning (Aslakson et al., 2014). The protocol included all four possible evidence sources- professional, lay, published and unpublished. The results are then proposed to be used to inform decision making around the inclusion of healthcare aids in complex interventions.

Lastly, Diouf et al. (2016) published an update of an environmental scan about training programs regarding 'shared decision making' (SDM) for health professionals, a model that promotes both health professionals and patients sharing the decision-making, as opposed to the former having complete autonomy (Diouf et al., 2016). In this update, the researchers accessed only published information, from professional (systematic reviews), grey (Google searches) and lay (social networks) viewpoints. The authors concluded that there continues to be large growth in the area of shared decision making, as seen through the large increase in SDM training programs. However, these training programs varied widely and were sparsely evaluated- two areas where there exists much room for improvement.

\subsection{How is an Environmental Scan Conducted?}

Before delving into the steps that take place in an environmental scan, it can be helpful to first get an idea of the overall picture (Figure 1). The first three steps, the why-who-what 
of the scan, involve a lot of planning and discussions, but are instrumental to conducting an effective scan. The fourth step, gathering information, is the most resource-heavy step as it involves looking for and obtaining relevant information, online and in person. The fifth and final step is to disseminate the information gathered in a way that the intended audience can understand and use. Just like the definition of an environmental scan, the steps involved are very flexible. The following are simply guidelines to keep in mind, not concrete steps.

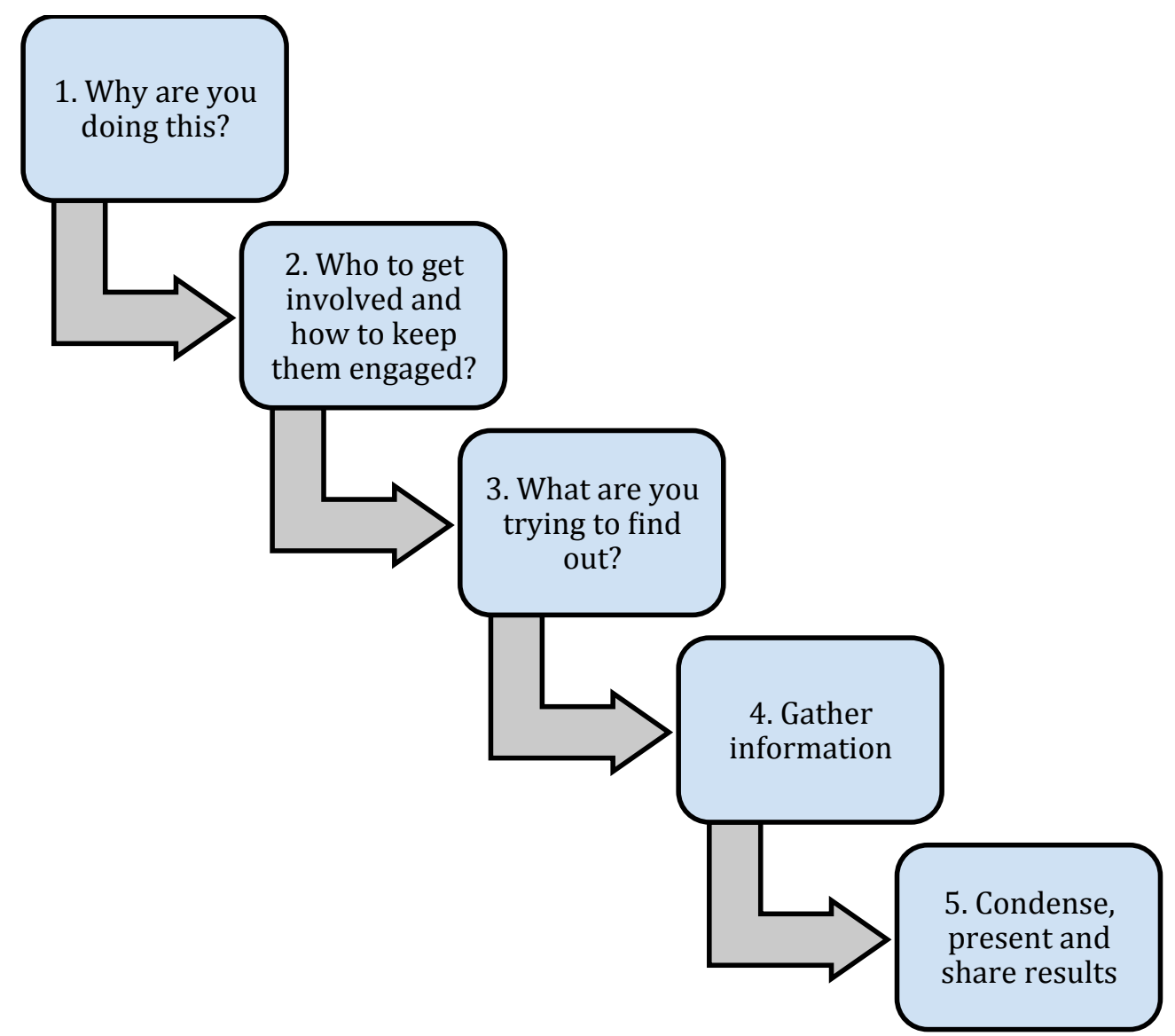

Figure 1: Bird's eye view of the environmental scan process.

\section{Step 1}

Although it may seem trivial, understanding precisely why an environmental scan is being done will streamline the rest of the process. For example, knowing the 'why' will help when deciding who to approach, which articles to include, and so forth. Figure 2 outlines three general processes that can assist in clarifying the purpose of your scan. Coming up with outlines of the questions that you are looking to find answers for (e.g. why are only $\mathrm{n} \%$ of a population undergoing breast cancer screenings) can help you and your team be on the 
same page regarding the purpose of the scan. Having your team do this step as individuals first and then in a group can assist in allowing for more productive group discussions since each member will have an idea of where they stand, as opposed to coming into a brainstorming session and attempting to understand the issue while others already have a solid understanding. Note that the questions do not have to be finalized at this stage, but rather a solid understanding of the general questions or topic is what is key.

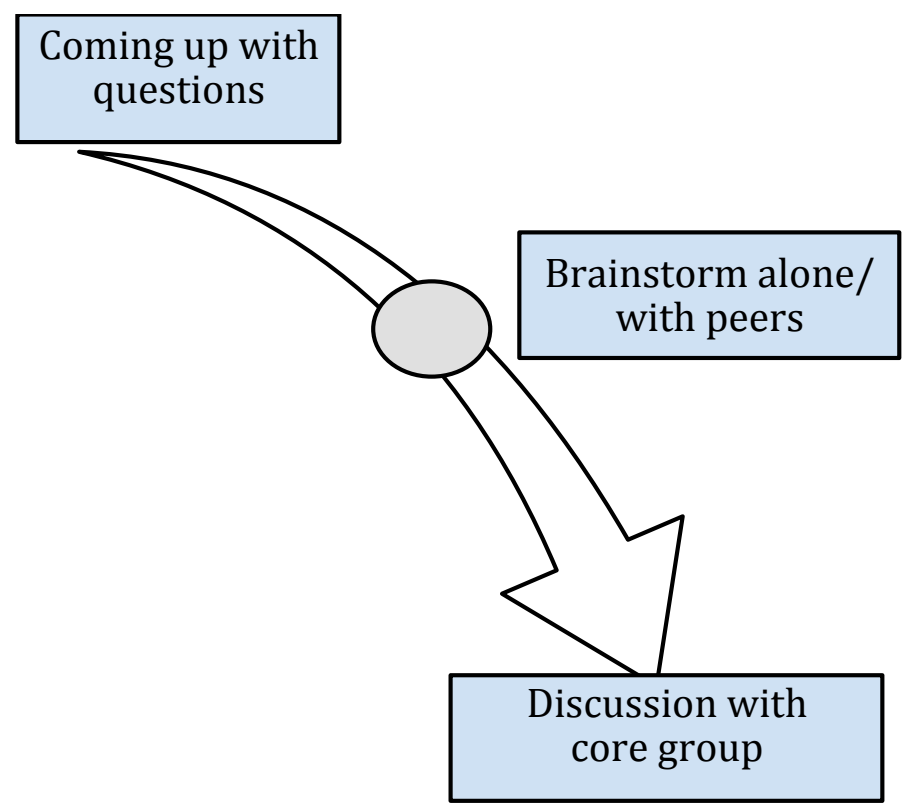

Figure 2: Define the purpose of the environmental scan.

\section{Step 2}

Approaching individuals relevant to the issue to gather information is one aspect that makes environmental scans different from other methodologies, such as literature reviews. This step lays the groundwork for when individuals will be approached later, in step 4. Before approaching individuals, it needs to be decided who will be approached. Three possible groups of people that can be approached are people with a shared interest in the issue, people who are impacted by the issue, and formal/informal leaders associated with the issue. Although people can be approached who do not fall into one of these categories, these can be good first steps. One option that can be further investigated is to go a step further and create a strategy to keep these stakeholders engaged during the study, if deemed beneficial. These steps are outlined in Figure 3.

\section{Step 3}

This stage is where the general questions outlined in step 1 need to be clarified and finalized. This is because once that is accomplished, then other details can be taken care of, namely, 


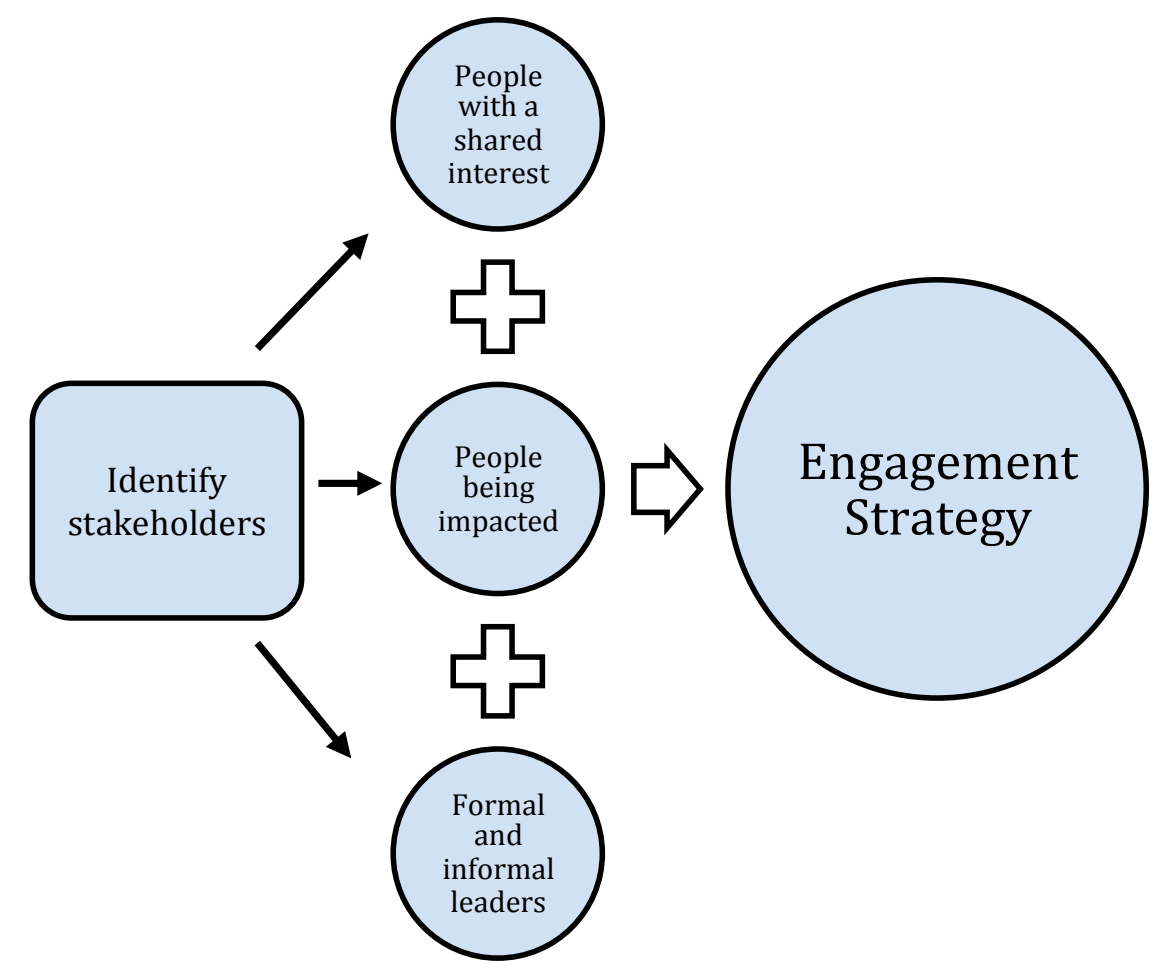

Figure 3: Identify stakeholders to develop engagement strategy.

what information is needed and where or from who that information can be obtained. For example, national databases, libraries and institutions are some areas to consider for information. Steps two and three might present some new information or an area where there is a lack of information. Due to this, it helps to go over the questions and make sure that they can still be addressed, or to tweak them accordingly. It is critical to be cognizant of "what you are trying to find out" while working on the other steps mentioned earlier (Figure 4).

\section{Step 4}

As mentioned earlier, this step is the most resource-heavy step, as it requires extensive and comprehensive information gathering. As seen in Figure 5, the four sources of information that can be delved into are: professional \& published, lay \& published, professional \& unpublished, and lay \& unpublished. Professional \& published information, which include peer reviewed scientific papers in academic journals and technical reports, can be found through systematic and grey literature reviews. More information on conducting reviews can be found elsewhere (Ahmed et al., 2016), and is outside the scope of this paper. Lay \& published information includes papers that have not been formally published or peer reviewed, such as association updates. This type of information can be found through a 


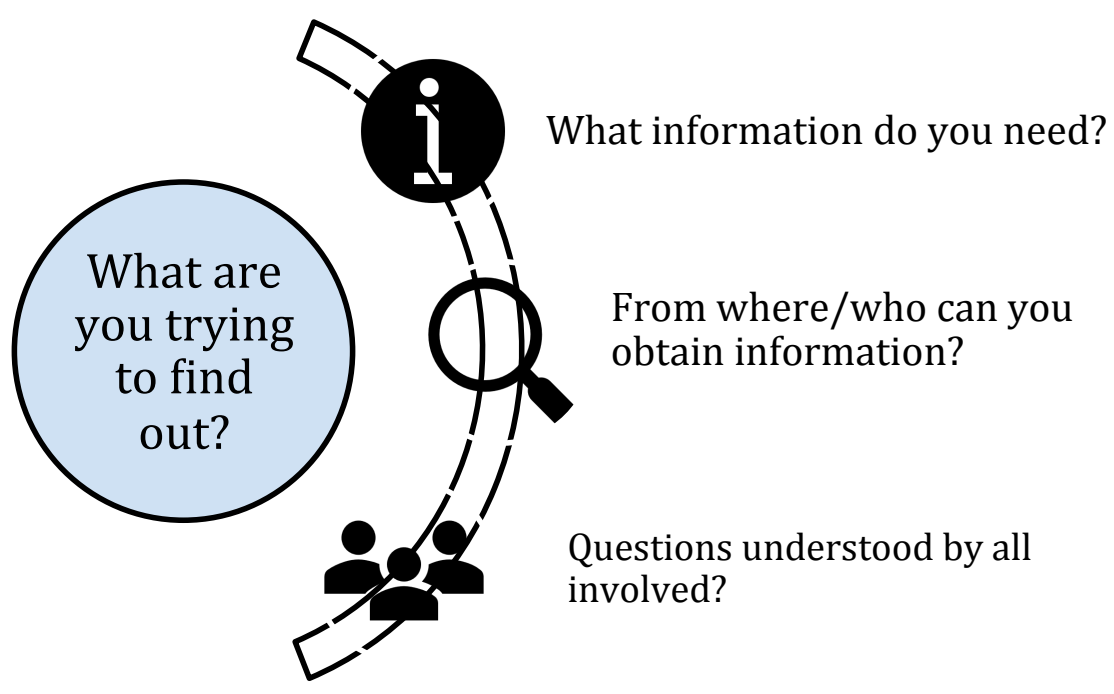

Figure 4: Create guiding questions for the environmental scan.

grey literature review, which is different from systematic reviews in the sense that papers excluded in systematic reviews can be included in grey literature reviews.

The reason grey literature reviews can be used for both professional and lay perspectives is that some institutions or leaders on specific issues who can be considered "experts" would fall into the professional category more than the lay one. The line between professional and layperson is not always concrete, thus conducting a grey literature review can help to find data in both categories. For a research paper, it may be necessary to acquire ethics approval for these next two steps as they include interviewing human subjects. Professional \& unpublished information can include presentations, talks, and other unwritten sources produced by experts and leaders. Lay \& unpublished information is gained from people not considered leaders or experts, but have important insights nonetheless. These can include people affected or at risk of being affected by the issue, for example. This information can be accessed through interviews, surveys and focus groups, to name a few methods.

\section{Step 5}

Consolidating all the information gathered in the previous step and disseminating it in a way that is appropriate to the intended recipients is crucial to achieve the desired effect of an environmental scan. Although the authors of the scan may have a solid understanding of the information, it needs to be shared with others who were not involved in the study, at a level that can be understood. When synthesizing the information gathered, it helps to remember what the purpose of the scan was - it will help in deciding which information to focus on or simply skim over. A sound methodological approach provides quality to the evidence generated.

Documenting the generated evidence in a comprehensive and systematic way is critical for further usage of the work. Dissemination is a very important component of the en- 


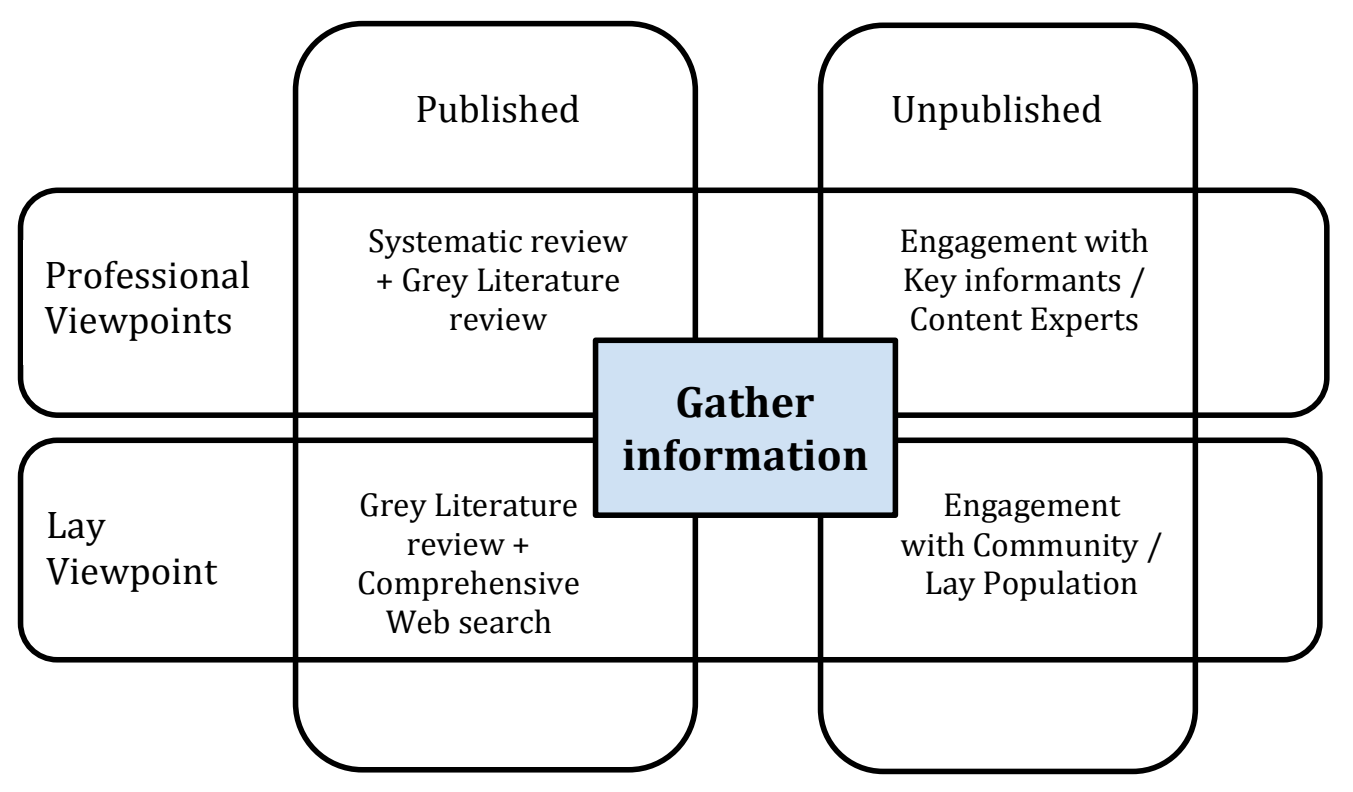

Figure 5: Sources of evidence. Adapted with permission from Aslakson et al. (2014).

vironmental scan, in which the information should be presented in a way that is easy to understand and easy to remember; this may differ depending on the audience. As a rule of thumb, figures and graphs are easier to digest and understand, as opposed to tables or paragraphs. Finally, incorporate the presentation material, and go into further details on relevant topics, in a report or a book, whatever method the recipients of the work would find beneficial. These points are represented in Figure 6.

Although the steps above outline the development of an environmental scan, it is important to remember one of the defining qualities of these tools: their flexibility. It is important to incorporate the specific issue into the scan by focusing on areas that may be of greater concern or more fruitful (e.g. for areas of new health research, there might be a gap in the professional and published perspectives, but prominent experts in the field who are doing cutting-edge research could provide valuable professional and unpublished information).

\section{Conclusion}

Overall, just like any other tool used for research, environmental scans have their benefits and challenges, as seen in Table 2. These tools hold a lot of potential as health research continues to expand and demands increasing amounts of data with dwindling resources. As seen through their recognition as a valuable tool (Graham et al., 2008), environmental scans are one tool that can help assess different health related issues in an effective manner since they don't require expensive technological instruments, but rather work ethic and methodologically clear strategies.

For students and researchers new to them, this paper serves as a roadmap to understanding and conducting environmental scans. Although the five steps outlined in this paper are very flexible, they are great points on which to design a scan. As part of the larger process 


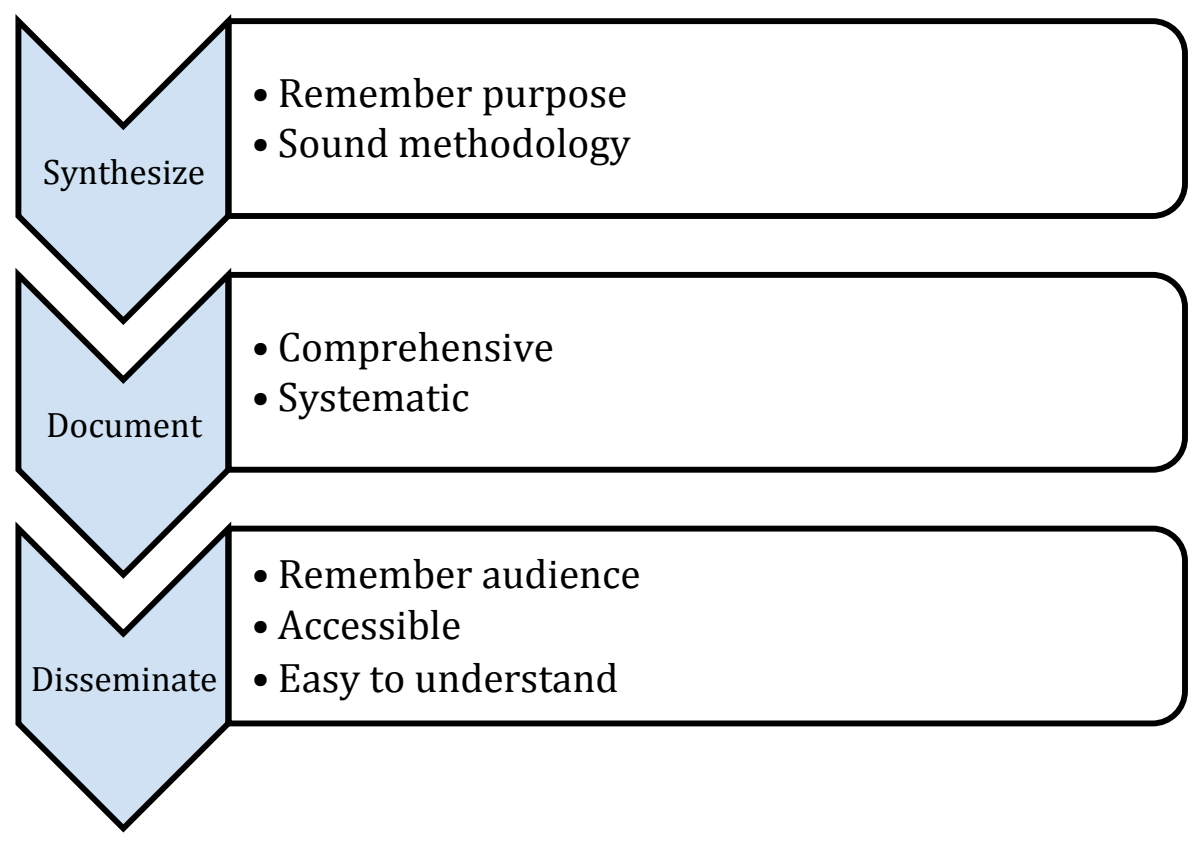

Figure 6: Synthesize, document and disseminate.

Table 2: Advantages and Challenges of using an Environmental Scan.

\section{Advantages}

Ability to change the scan to align with specific contexts and available resources due to the lack of a standardized method of conducting environmental scans

Draws on both published and unpublished information to decrease chances of overlooking relevant information

Incorporates both professional and lay perspectives to provide a healthy balance between scientific rigour and public opinion

\section{Challenges}

Inability to compare different scans due to a lack of a standardized method of conduct-

ing environmental scans

Resource/time heavy \& possibility of gathering incorrect and conflicting information

Difficulties in reconciling information may arise if professional perspectives conflict with lay/public perspectives

of assessing the context of a health issue, this tool can help researchers in doing their due diligence around an issue before dedicating countless time and resources to one.

\section{Declarations}

Funding: None.

Conflict of interest: The authors declare that they have no conflict of interests. 
Ethical approval: Not applicable.

\section{References}

Ahmed S, Vaska M, Turin TC (2016). "Conducting a Literature Review in Health Research: Basics of the Approach, Typology and Methodology." Journal of National Heart Foundation of Bangladesh, 5(2), 44-51.

Albright KS (2004). "Environmental scanning: radar for success." Information Management, 38(3), 38.

Aslakson RA, Schuster ALR, Miller J, Weiss M, Volandes AE, Bridges JFP (2014). "An environmental scan of advance care planning decision AIDS for patients undergoing major surgery: a study protocol." The Patient, 7(2), 207-217. doi:10.1007/s40271-014-0046-3.

Canadian Agency for Drugs and Technologies in Health (May, 2015). "Environmental Scan Process [Accessed on 2018 Jan 13]. Available from: https://www.cadth.ca/environmental-scanning." URL https://www.cadth.ca/sites/ default/files/pdf/ES\%20External\%20Audience\%20Process\%20Doc.pdf.

Choo CW, et al. (2001). "Environmental scanning as information seeking and organizational learning." Information Research, 7(1), 7-1.

Diouf NT, Menear M, Robitaille H, Guérard GP, Légaré F (2016). “Training health professionals in shared decision making: Update of an international environmental scan." Patient Education and Counselling, 99(11), 1753-1758.

Glasziou P, Altman DG, Bossuyt P, Boutron I, Clarke M, Julious S, Michie S, Moher D, Wager E (2014). "Reducing waste from incomplete or unusable reports of biomedical research." The Lancet, 383(9913), 267-276. doi:10.1016/S0140-6736(13)62228-X.

Graham P, Evitts T, Thomas-MacLean R (2008). "Environmental scans: How useful are they for primary care research?" Canadian Family Physician, 54(7), 1022-1023.

Morrison JL (1992). "Environmental scanning." In M Whitely, J Porter, R Fenske (eds.), A primer for new institutional researchers, chapter Environmental scanning, pp. 86-99. The Association for Institutional Research, Tallahassee, Florida.

Rowel R, Moore ND, Nowrojee S, Memiah P, Bronner Y (2005). "The utility of the environmental scan for public health practice: lessons from an urban program to increase cancer screening." Journal of the National Medical Association, 97(4), 527.

BRF www.jBiomedAnalytics.org 